\title{
A Study on Histogram Moments and their Application to Image Thresholding
}

\author{
Salah Ameer \\ Faculty of Applied Science and Technology, Humber College, Canada
}

\begin{abstract}
Histogram moments have been widely investigated for image thresholding. This paper proposes several novel schemes in this area. In some of the proposals, the idea is simply to match a moment from the original image (calculated from the histogram) to a corresponding moment of the thresholded image. In other proposals, the threshold is the one optimizing a specific moment. Comparative results with Otsu shows the effectiveness of the proposed schemes.
\end{abstract}

\section{Introduction}

Image thresholding has been widely investigated due to its vital role in many applications and one of the effective methods for image segmentation. Various schemes have been proposed in the literature, a good review can be found in [1].

The histogram plays a crucial role in many of these schemes. In general, the histogram is used as an approximation to the probability density function [2-3]. In these cases and their extensions, the threshold is selected as a solution to an optimization problem for some objective function dependent on features extracted from the histogram.

The aforementioned schemes can be generalized to multi-level thresholding as in [4]. However, the computational price is too high.

An interesting scheme to preserve the moments was proposed by [5]. For binary thresholding, the first three moments of the thresholded image have to be equal to those of the original image.

A higher dimensional histogram can be constructed using the local variance [6].

This research proposes few formulations that exploit the use of different moments deducted from the 1D histogram. In general, the optimum threshold(s) are the ones producing a moment match (to that of the original) or the moment attains its optimum value at these threshold(s).

\section{Preliminaries}

The following symbols are adopted in all subsequent sections:

$g_{i}=$ The $i^{\text {th }}$ gray level in the image .

$h=$ histogram.
$H$ = Cumulative histogram.

$L=$ number of gray levels in the image.

$N=$ Number of pixels in the image.

$X_{O}=$ Feature from the original image.

$X_{T}=$ Feature from the thresholded image.

$T=$ Threshold.

$s_{<T}=$ Size of gray levels below the threshold.

$a_{<T}=$ Average of gray levels below the threshold.

$V_{<T}=$ Variance of gray levels below the threshold.

$s_{>_{T}}=$ Size of gray levels above the threshold.

$a_{>_{T}}=$ Average of gray levels above the threshold.

$V_{>T}=$ Variance of gray levels above the threshold.

$m_{p}=\sum_{i} h_{i}\left|g_{i}-a_{o}\right|^{p}$

$n_{p}=\sum_{i} h_{i}\left|\frac{g_{i}-a_{O}}{\sqrt{V_{O}}}\right|^{p}$

Without loss of generality, the gray levels are normalized to the interval $[0,1]$. Also, the histogram is normalized such that its sum is equal to one.

For the purpose of comparing the performance of the thresholding schemes, many evaluation criteria have been suggested [1].

A simple evaluation scheme, Ratio Error, is proposed given by:

$D S M=\left|\frac{\# F_{T}}{\# B_{T}}-\frac{\# F_{G}}{\# B_{G}}\right|$

where, $F_{G}$ is the foreground of ground truth, $B_{G}$ is background of ground truth, $F_{T}$ is the foreground of thresholded image, $B_{T}$ is the background of thresholded image and \# is the cardinality of the set.

Obviously, a value of 0 indicates best match and the higher the value the more the thresholded image is away from the ground truth image. Unfortunately, the measure is not normalized, however its simplicity is obvious.

\section{Spread Function}

Each bin in the histogram (corresponding to gray level $g_{i}$ ) is replaced by a Gaussian function having an amplitude of $\left(\mathrm{h}_{i}\right)$ centered at $g_{i}$ and a variance equal to that of the original image divided by L (number of gray levels). The resultant histogram will be the sum of all the Gaussian functions. Mathematically,

$h^{\prime}(x)=\sum_{i} h_{i} e^{-\left(x-g_{i}\right)^{2} /\left(\frac{2 V_{O}}{L}\right)}$ 
Intuitively, the best thresholds would be the minima of the resultant histogram. The global minimum can be picked (reported in the experimental section), however, all minima should be picked for better performance as concluded from many tests.

After performing the multi-level thresholding, the procedure can be repeated untill we reach the no change state (an iteration resulting in no change in terms of number of gray levels).

The division by $\mathrm{L}$ inherently means an equal split of the power between the bins since no aposteriori information is available. The ambiguity is wheather to use $\mathrm{L}$ in every iteration or use the number of levels from the previous iteration. The former produces more number of gray levels at the output, while the latter tends to have inferior binarization quality.

\section{Moments}

In this section, different moments are investigated to obtain the best threshold. All of the proposed schemes implement exhaustive search to find the optimum threshold.

\subsection{Minimum Moment MM}

The optimum threshold is where a certain moment attains its lowest value,

$$
T_{M M}=\arg \left\{\min _{x}\left(\sum_{1}^{L}\left|g_{i}-g_{x}\right|^{p} h_{i}\right)^{1 / p}\right\}
$$

Unfortunately, its ambiguous as which moment is more important. In general, positive values of $p$ are smoth and result in a single threshold. However, each image will respond differently to a specific value of $p>0$.

On the otherhand, negative values of p results in many values for $\mathrm{T}_{\mathrm{MM}}$, see Eq.(3), leading to a similar iteration paradigm to that of the previous section, Spread Function.

A mixure can be implemented, however, a careful selection of moments should be performed first. Fractional moments may be considered as well.

\subsection{Maximum Central Moment MCM}

The hypothesis here is that the optimum threshold is the one producing the highest central moment,

$$
T_{M C M}=\arg \left\{\max _{T}\left(s_{<T}\left|a_{<T}-a_{O}\right|^{p}+s_{>T}\left|a_{>T}-a_{O}\right|^{p}\right)^{1 / p}\right\}
$$

In other words, the best thresholded image is the one producing values as distant as possible from the mean and each other.

As expected, higher values of $\mathrm{p}$ can result in producing many maxima, and hence iteration may be required.

In addition, a specific moment may produce the best result to an image but performs poorly on another.

\subsection{High Central Moment HCM}

As a modification to the previous sub-section, we can merge the gray levels producing a central moment lower than that of the original Image. Unfortunately, the final image will have high number of gray levels.

\section{Moment Matching}

The matching scheme can be transferred into a root finding algorithm, in other words, find the solution to the equation, in a similar fashion to Eq. (4)

$$
s_{<T}\left|a_{<T}-a_{O}\right|^{p}+\left(1-s_{<T}\right)\left|a_{>T}-a_{O}\right|^{p}=m_{p}
$$

Fortunately we can write ao, the image average as

$$
a_{o}=s_{<T} a_{<T}+\left(1-s_{<T}\right) a_{>T}
$$

Plugging Eq.(6) into Eq.(5) and utilizing the fact that $a_{>T}>a_{O}>a_{<T}$, we have (subscript for $s$ is dropped for simplicity)

$$
\left(a_{>T}-a_{<T}\right)^{p}\left\{s(1-s)^{p}+(1-s) s^{p}\right\}=m_{p}
$$

Eq.(7) can now be solved for s (number of dark pixels). After finding s, we can find the threshold through the cumulative histogram $\mathrm{H}$ via

$$
T=\arg \left\{\min _{i}\left|H_{i}-s\right|\right\}
$$

It should be noted that Eq.(7) needs further modification if the value of $p$ was a negative value or noninteger.

\subsection{Roots Matching RM}

Unfortunately, Eq.(7) may not have a soltion. A possible remedy would be to solve, normalizing thresholded image to $[0,1]$ (subscript for $\mathrm{s}$ is dropped for simplicity)

$$
T_{R M}=\arg \left\{\min _{s}\left(s(1-s)^{p}+(1-s) s^{p}-m_{p}\right)^{2}\right\}
$$

Unfortunately, the thresholded image is at least trilevel. Combining more than one moment requires extra work on the weighting scheme and equal weights did not perform well experimentally. However, the solution does not require exhaustive search.

\subsection{Divergence from Cumulative Histogram DCH}

Another remedy is to solve Eq.(7) for each possible threshold (exhaustive search). The optimum threshold is the gray level having its cumulative histogram value closest to its solution, or 
$T=\arg \left\{\min _{g}\left|H_{g}-s_{g}\right|\right\}$

\subsection{Merging the Ends ME}

A merging scheme may be implemented using the solutions for $\mathrm{n}$ resulting from Eq.(7). The two roots are ordered, picking the lowest and highest possible solutions. These two solutions are compared to the cumulative histogram $\mathrm{H}$ to find the corresponding gray levels. Merging is then performed from these gray levels to the appropriate end ( 0 or 1$)$. However, the final image will have high number of gray levels.

\subsection{Moment around the threshold MT}

Defining the moment around a threshold $\mathrm{g}_{\mathrm{t}}$ as

$t_{p}=\sum_{i} h_{i}\left|g_{i}-g_{t}\right|^{p}$

Following the same layout as that of Eq.(5), the objective is to minimize the following

$\left|\left\{s_{<T}\left|a_{<T}-g_{t}\right|^{p}+\left(1-s_{<T}\right)\left|a_{>T}-g_{t}\right|^{p}\right\}^{\frac{1}{p}}-\left(t_{p}\right)^{\frac{1}{p}}\right|$

The value(s) of $g_{t}$ producing a minimum in Eq.(12) is selected as a threshold.

An alternative to Eq.(12) is to treat $\mathrm{n}_{<\mathrm{T}}$ as a variable and solve for it, resulting in:

$s_{<T}=\frac{t_{p}-\left|a_{>T}-g_{t}\right|^{p}}{\left|a_{<T}-g_{t}\right|^{p}-\left|a_{>T}-g_{t}\right|^{p}}$

Any value of $g_{t}$ that does not produce a solution in $(0,1)$ will be merged with its neighbour.

Iteration is required to both Eq.(12) and Eq.(13) to reduce the number of gray levels in the resultant image.

\section{Normalized Moments}

In many application, the random variables are normalized using their mean and standard deviation as

$y=\frac{x-a_{x}}{\sqrt{V_{x}}}$

Normalizing the thresholded image to [0 1], we have

$a_{T}=s_{>T}$

$V_{T}=s_{<T}\left(0-a_{T}\right)^{2}+s_{>T}\left(1-a_{T}\right)^{2}=s_{<T} S_{>T}$

Eq.(14) can now be utilized in many schemes as described in the following subsections.

\subsection{Normalized Trimming NT}

In this scheme, the tails beyond a normalized value of 1 (right tail) or -1 (left tail) are merged. Iterations are needed. The thresholded image will have a high number of gray levels.

\subsection{Highest Normalized Moment HNM}

The idea is similar to that of Eq.(4), with the incorporation of Eq.(14) - Eq.(16), we have

$T_{H N M}=\arg \left\{\max _{T}\left(s_{<T}\left|\frac{-s_{>T}}{\sqrt{s_{>T} s_{<T}}}\right|^{p}+s_{>T}\left|\frac{1-s_{>T}}{\sqrt{s_{>T} s_{<T}}}\right|^{p}\right)^{1 / p}\right\}$

\subsection{Matching Normalized Moments MNM}

In this scheme, the best threshold is the one producing a thresholded image with a normalized moment closest to that of the original image.

Unfortunately, each image produces the best match for a different value of $\mathrm{p}$. Hence, a more thorough investigation is needed.

\subsection{Normalized Roots Matching NRM}

The moment of Eq.(17) is compared to that of the original image, with the use of Eq.(14) - Eq.(16), we have (subscript for $\mathrm{s}$ is dropped for simplicity)

$T_{N R M}=\arg \left\{\min _{s}\left(s\left(\frac{1-s}{s}\right)^{p / 2}+(1-s)\left(\frac{s}{1-s}\right)^{p / 2}-n_{p}\right)^{2}\right\}$

Obviously no exhaustive search is needed, however, the performance is inferior.

\section{Zero Moment}

In reference to Eq.(3), Eq.(4), Eq.(5) and their normalized version (incorporating Eq.(14)), we can use $p$ $=0$. However, the results will be trivial as all thresholds are possible. An intuitive approach is to use the derivative with respect to $\mathrm{p}$, resulting in the log moments. The new proposed schemes are minimum log moment MinL, maximum log moment MaxL, and matching log moment MatM, given by

$$
\begin{aligned}
& T_{\text {MinL }}=\arg \left\{\min _{x}\left(\sum_{1}^{L} \log \left(\left|g_{i}-g_{x}\right|\right) h_{i}\right)\right\} \\
& T_{M a x L}=\arg \left\{\max _{T}\left(s_{<T} \log \left(\left|a_{<T}-a_{O}\right|\right)+s_{>T} \log \left(\left|a_{>T}-a_{O}\right|\right)\right)\right\}(20) \\
& s_{<T} \log \left(\left|a_{<T}-a_{O}\right|\right)+s_{>T} \log \left(\left|a_{>T}-a_{O}\right|\right)=\sum_{i} h_{i} \log \left(\left|g_{i}-a_{o}\right|\right)
\end{aligned}
$$

Some values need to be removed to avoid $\log (0)$. 


\section{Experimental Results}

The performance of the suggested schemes based on Eq. $(2-18)$ are reported. No comparison will be given to schemes that can only produce a multilevel thresholded image. In addition, schemes that can have both multilevel and bilevel thresholded image will be reported as bilevel and compared with Otsu method due to its popularity. Fig. 1 illustrate the original images and their ground truth together with Otsu result.

Fig. 2 shows the multilevel thresholding results for the spread function scheme, Eq.(2). The thresholding using the global minimum has inferior performance and hence not reported.
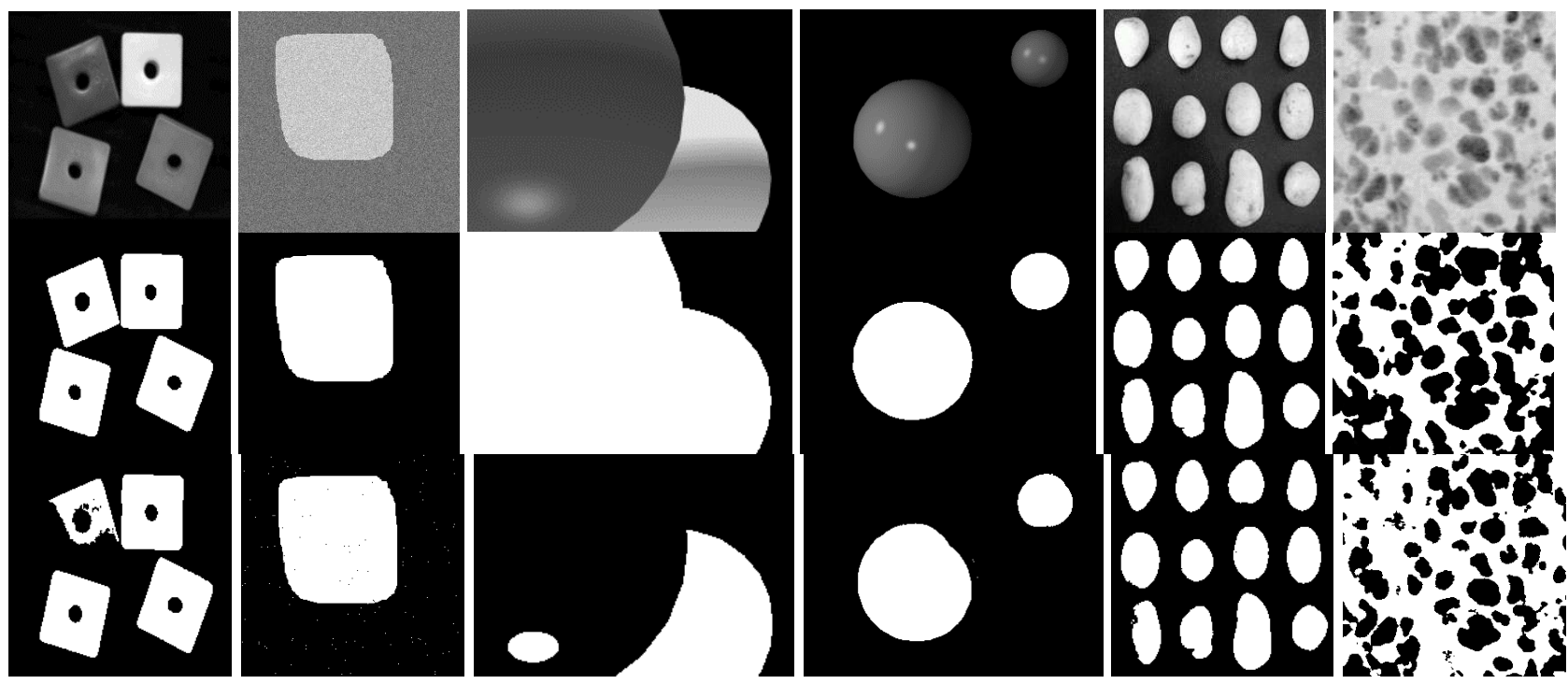

Fig. 1: (Top to buttom) Test images, ground truth, and Otsu.
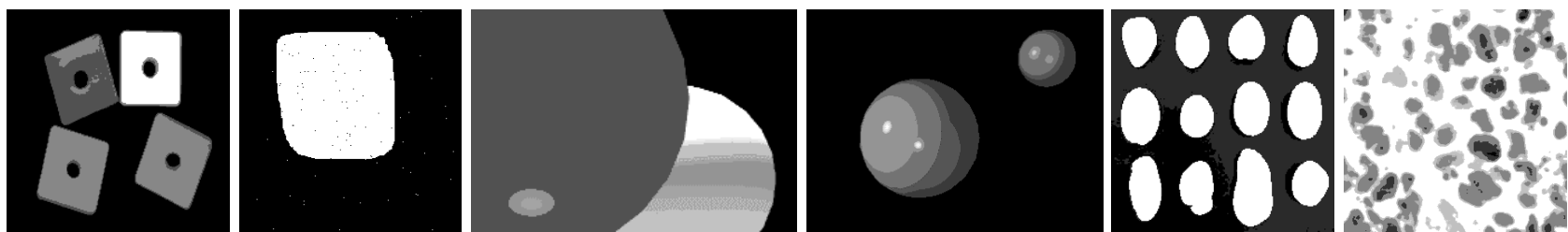

Fig. 2: Resultant multilevel thresholding images using spread function, Eq.(3). Number of levels (left to right): 5,2,11,12,3,7

Fig. 3 shows the results for the minimum moment scheme, using global minimum of Eq.(3), with p values of 1 and 2 .

Fig.4 shows the results for the maximum central moment scheme with $\mathrm{p}$ values of 1 and 2, Eq.(4).
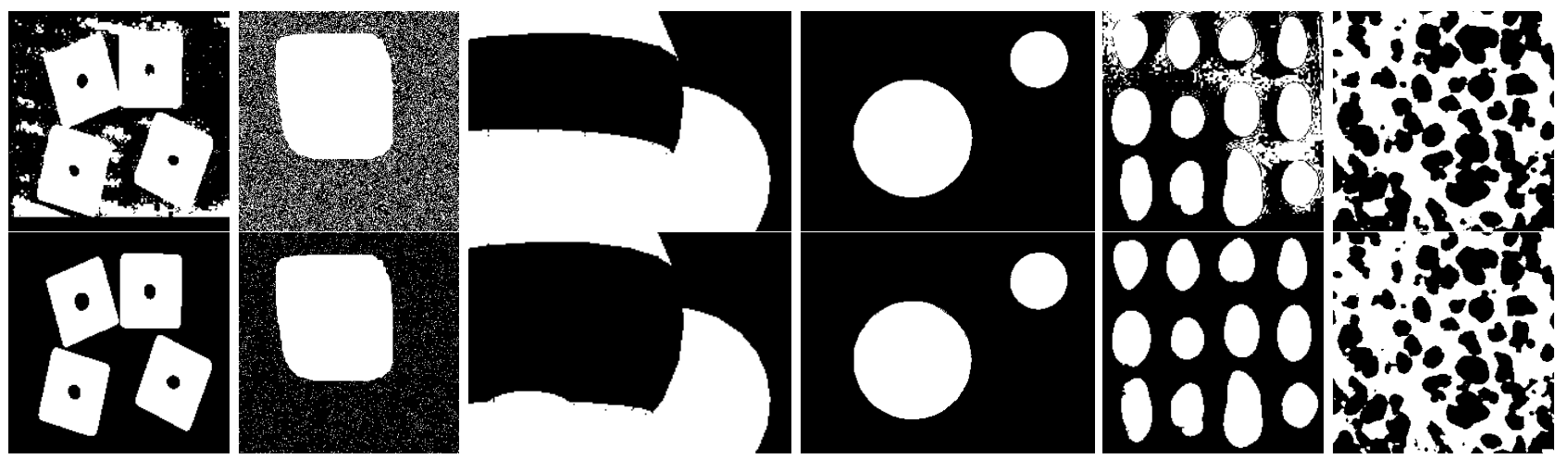
Fig. 3: Results for minimum moment scheme, Eq.(3). (Top to buttom) p =1, 2 .
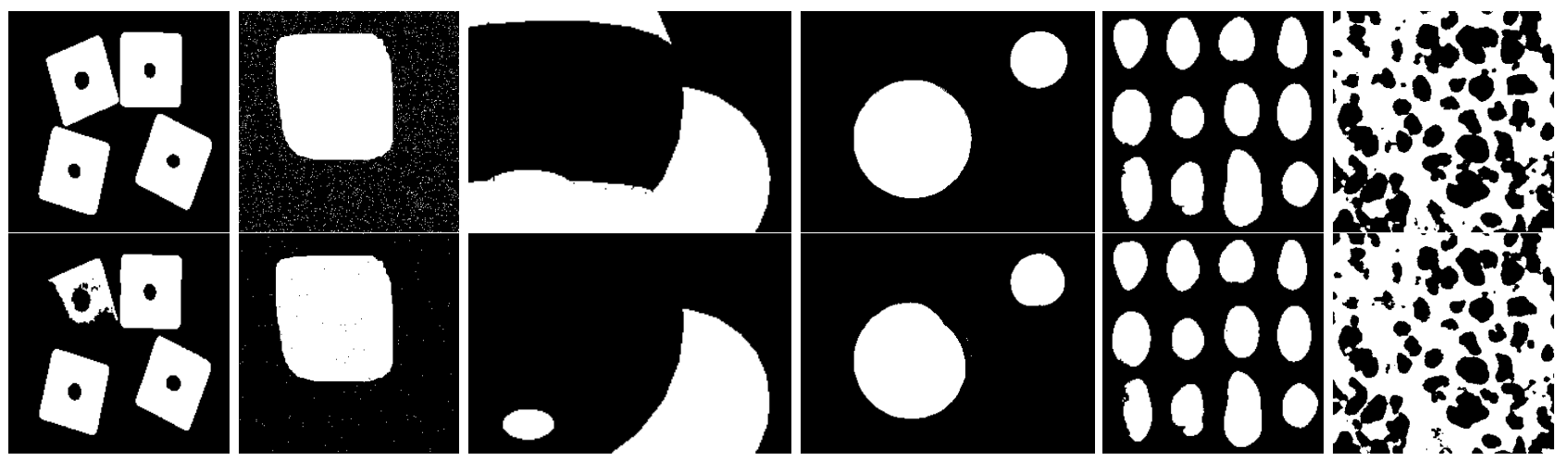

Fig. 4: Results for maximum central moment scheme, Eq.(4). (Top to buttom) p=1, 2.
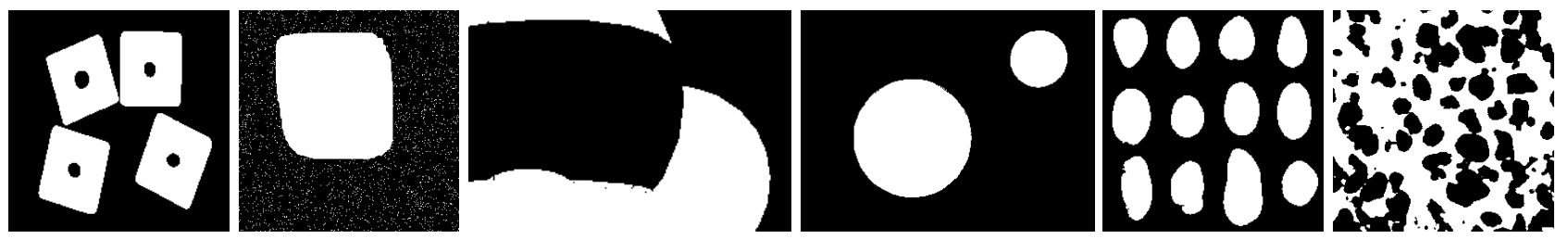

Fig. 5: Results for high central moment scheme, $p=1$.

Fig.6 shows the results for the roots matching scheme, Eq.(9).

Fig.7 shows the results for the DCH scheme, Eq.(10). More than one iteration is required to get the binary image, typically $2-3$.

Fig.8 shows the implementation of Eq.(12). Fig.9 shows the implementation of Eq.(13).
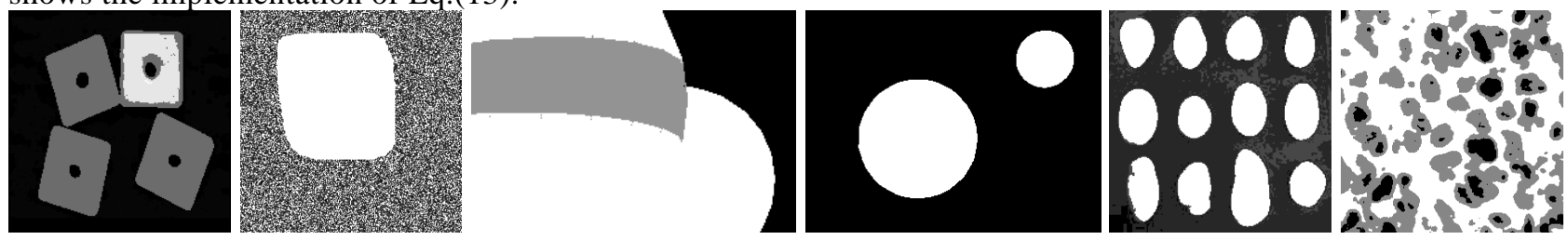

Fig. 6: Results for roots matching scheme. (left to right) $p=16,1,88,91,54,1$ and levels = 5, 3, 3, 2, 4, 3 .
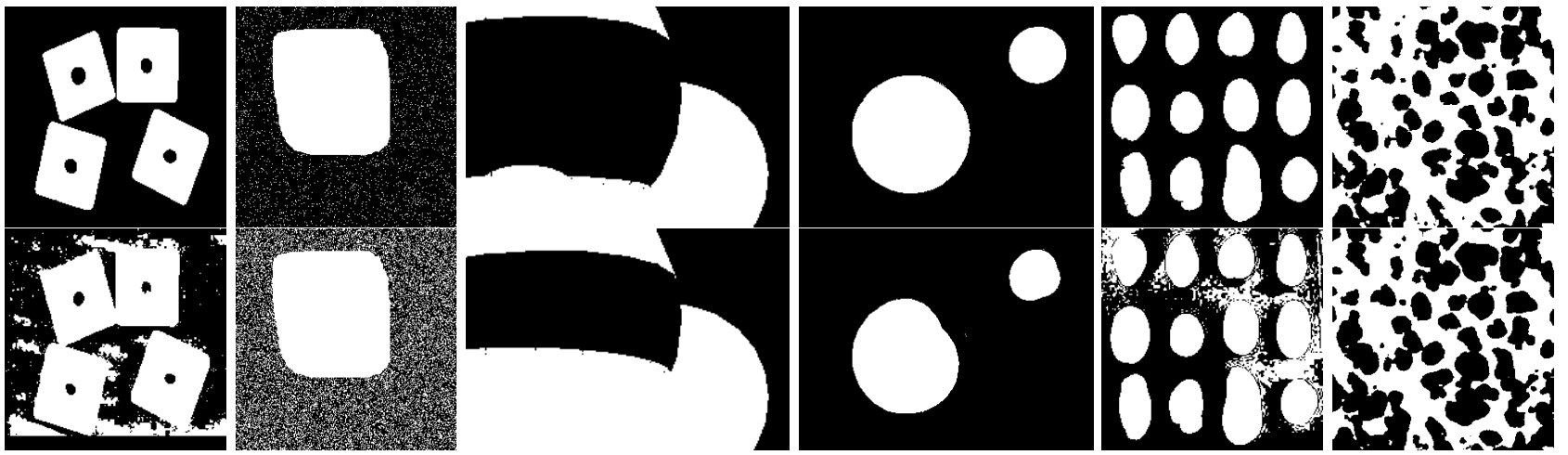

Fig. 7: Results for DCH scheme, Eq.(10). (Top to buttom) p=1, 2. 

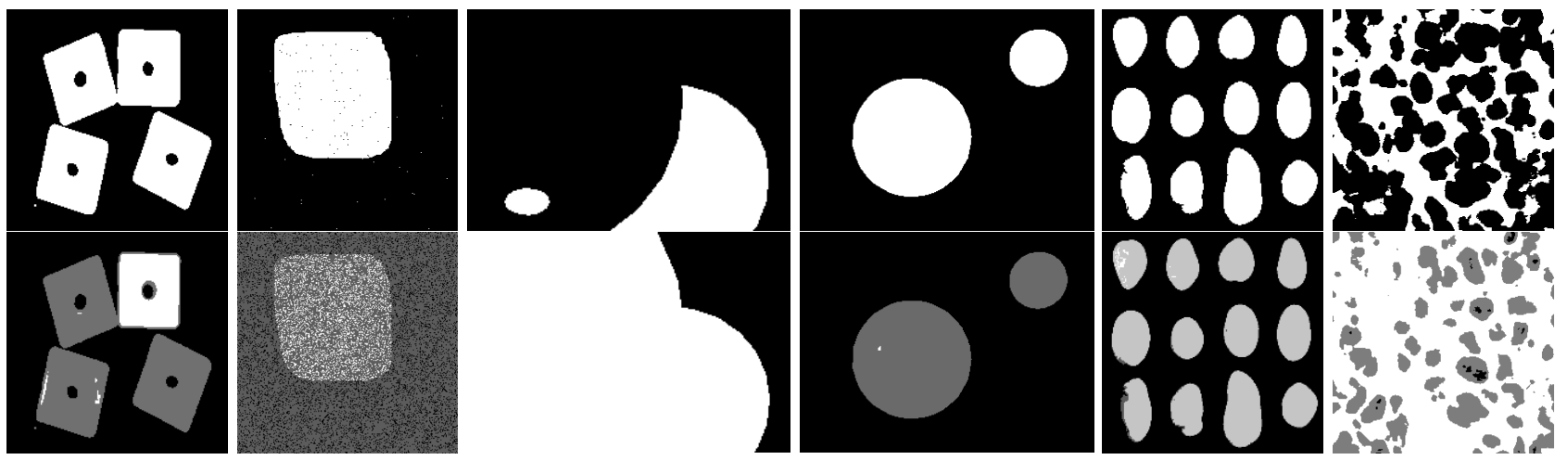

Fig. 8: Results for the implementation of Eq.(13). (Top to buttom) $p=0.5,1$.
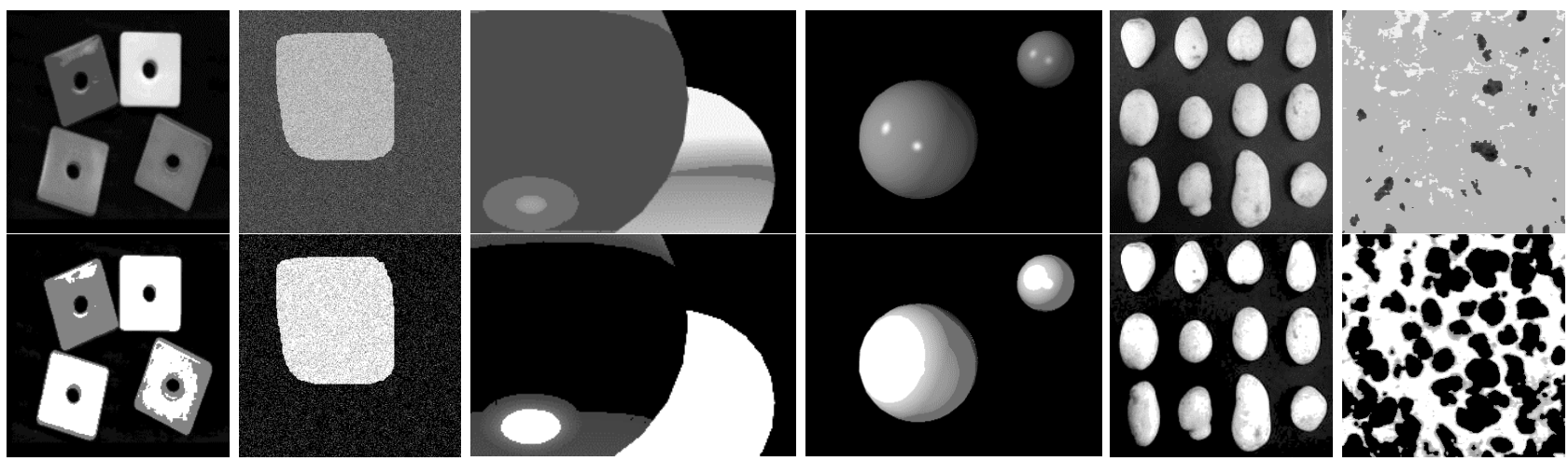

Fig. 9: Results for the implementation of Eq.(14). (Top to buttom) $p=0.5,2$.
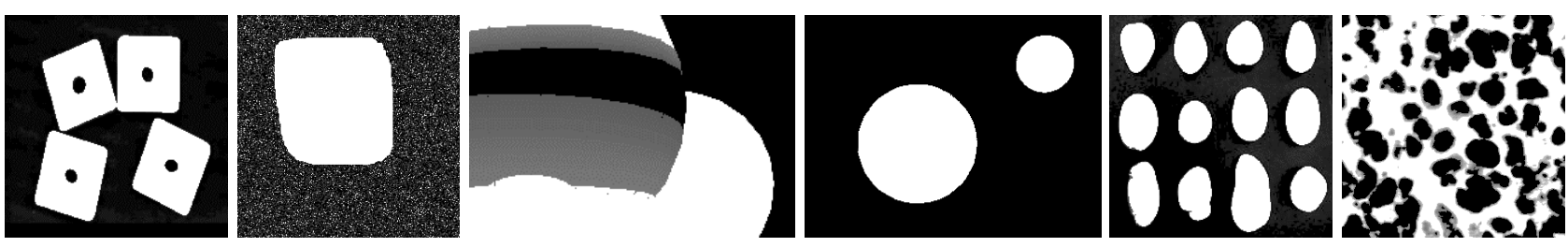

Fig. 10: Results for merging the ends scheme $(p=1)$. (left to right) levels/iterations =33/34, 27/12, 15/15, 16/23, 54/16, 27/10.

The zero moment formulatios given by Eq.(19), Eq.(20), and Eq.(21) are reported in Fig.12 - 14 respectively. As expected, the results of Fig.14 are multi-level.

The RE, Eq.(1), is used for evaluation. Table 1 lists the values for Otsu, MM (Fig.3), MCM (Fig.4), MinL (Fig.12), and MaxL (Fig.13).
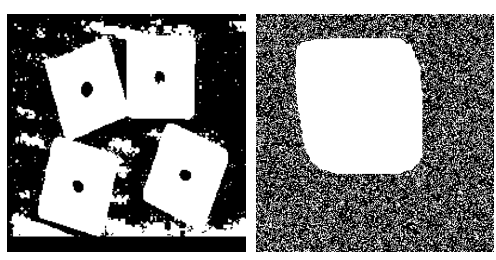
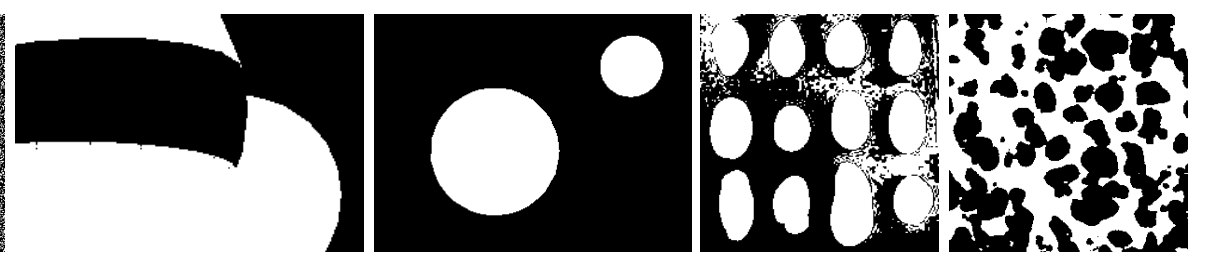

Fig. 11: Results for the implementation of Eq.(17), $p=1$.
Interestingly, the MCM scheme with $\mathrm{p}=2$ is an equivalent formulation to Otsu (1975). A closer look at Table 1 reveals that better performance can be optained with different values of $p$. The first central moment seems to have a slight improvement, however, it is image dependant. 

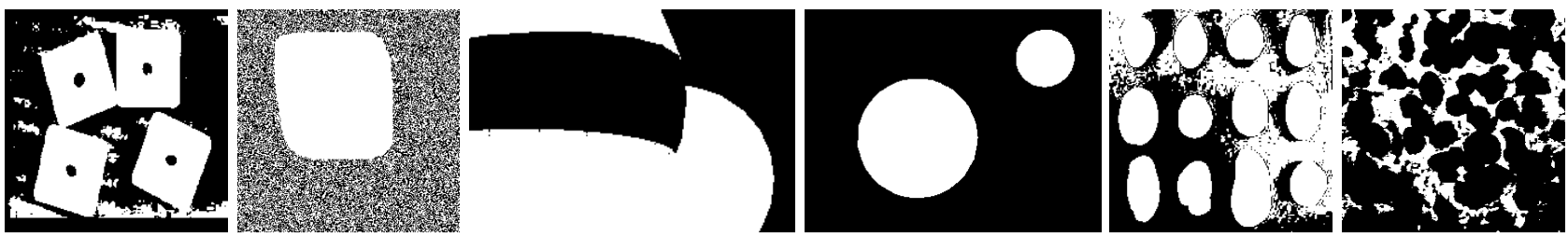

Fig. 12: Results for the implementation of Eq.(19), MinL.
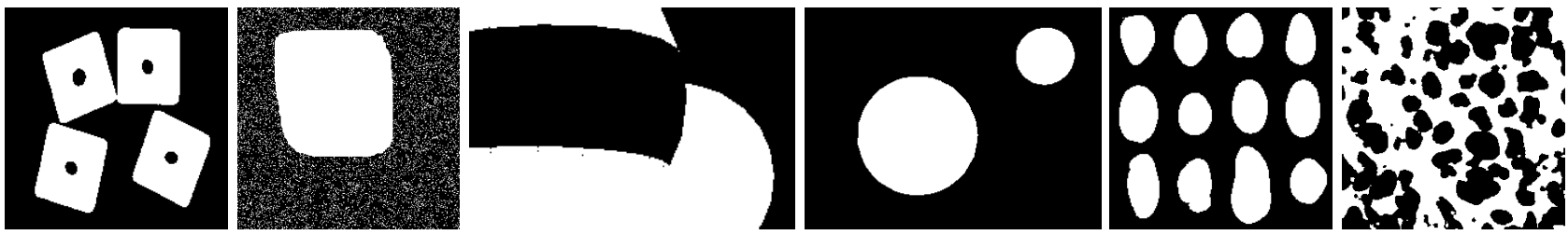

Fig. 13: Results for the implementation of Eq.(20), MaxL.
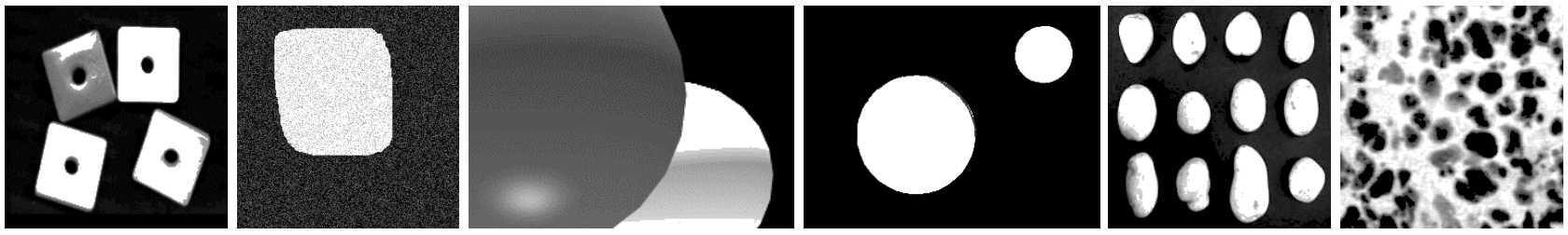

Fig. 14: Results for the implementation of Eq.(21), MatL. Levels/iteration (left to right): 100/6, 88/4, 157/3, 30/11, 147/6, 76/5.

Table 1: RE values for OTSU and some of the proposed schemes.

\begin{tabular}{|c|c|c|c|c|c|c|}
\hline Image/Method & 1 & 2 & 3 & 4 & 5 & 6 \\
\hline Otsu & 0.104 & 0.003 & 3.775 & 0.032 & 0.045 & 0.729 \\
\hline $\mathbf{M M}(-1)$ & 3.271 & 1.162 & 0.041 & 0.000 & 0.610 & 0.648 \\
\hline MM(1) & 0.401 & 0.632 & 2.978 & 0.000 & 0.393 & 0.051 \\
\hline MM(2) & 0.007 & 0.063 & 3.490 & 0.004 & 0.014 & 0.347 \\
\hline $\operatorname{MCM}(-2)$ & 0.072 & 0.386 & 3.156 & 0.000 & 0.084 & 0.170 \\
\hline MCM(1) & 0.007 & 0.063 & 3.490 & 0.004 & 0.014 & 0.347 \\
\hline MCM(2) & 0.104 & 0.003 & 3.775 & 0.032 & 0.045 & 0.729 \\
\hline MinL & 0.322 & 1.314 & 2.978 & 0.000 & 0.881 & 0.574 \\
\hline MaxL & 0.032 & 0.229 & 3.228 & 0 & 0.005 & 0.234 \\
\hline
\end{tabular}

\section{Conclusion and Future Work}

New algorithms for image thresholding has been proposed in this work using simple moment computations from the histogram.

Results are promising, however, more test images are needed to explore the limits of the proposed schemes. The domain of application for each of the proposed schemes as well as their extension to higher dimensional histograms are currently under investigation.

Of special interest is the root matching schemes, Eq.(9) and Eq.(18), where the threshold can be found without an exhaustive search. More research is needed to find the optimum $p$ value for each image or maybe a single value of $p$ for a specific image domain.

More investigation is needed to control the number of thresholds. Suggestions can be weighted average of some moments, or combining several schemes.
A better decision making on whether to pick the global optimum or some of the local optima or some weighted average of the moment values at different gray levels.

\section{References}

[1] M. Sezgin, and B. Sankur, "Survey Over Image Thresholding Techniques And Quantitative Performance Evaluation". J. Electronic Imag., 2004, 13: pp 146-165. DOI: 10.1117/1.1631315.

[2] J. Kapur, P. Sahoo and A. Wong, "A New Method For Gray-Level Picture Thresholding Using The Entropy Of The Histogram". Comput. Vis. Graph. Image Process., 1985, 29: pp 273-285. DOI: 10.1016/0734189X(85)90125-2.

[3] Otsu, N., 1979. A threshold selection method from gray-level histograms. IEEE Trans. Syst. Man Cybernet., 9: 1979, pp 62-66. 
[4] D. Liu, and J. Yu, 2009. Otsu method and k-means. Proceedings of the 9th International Conference on Hybrid Intelligent Systems, IEEE Xplore Press, Shenyang, China, 2009, pp: 344-349.

[5] W. Tsai, "Moment-Preserving Thresholding: A New Approach. Computer vision". Graphics, and image processing 29, 1985, pp 377-393.

[6] X. Zheng, H. Ye, and Y. Tang, "Image Bi-Level Thresholding Based on Gray Level-Local Variance Histogram". Entropy, 19, 191; 2017, doi:10.3390/e19050191, pp 1-8. 